\title{
Erratum: Scaled momentum spectra in deep inelastic scattering at HERA
}

\section{ZEUS collaboration}

E-mail: tobias.haas@desy.de

ERrATUM to: JHEP06(2010)009

The following is the correct list of all authors and affiliations for the ZEUS collaboration.

H. Abramowicz ${ }^{44, a d}$, I. Abt ${ }^{34}$, L. Adamczyk ${ }^{13}$, M. Adamus ${ }^{53}$, S. Antonelli ${ }^{4}$, P. Antonioli ${ }^{3}$, A. Antonov ${ }^{32}$, M. Arneodo ${ }^{49}$, V. Aushev ${ }^{26, y}$, Y. Aushev ${ }^{26, y}$, O. Bachynska ${ }^{15}$, A. Bamberger ${ }^{19}$, A.N. Barakbaev ${ }^{25}$, G. Barbagli ${ }^{17}$, G. Bari ${ }^{3}$, F. Barreiro ${ }^{29}$, D. Bartsch ${ }^{5}$, M. Basile ${ }^{4}$, O. Behnke ${ }^{15}$, J. Behr ${ }^{15}$, U. Behrens ${ }^{15}$, L. Bellagamba ${ }^{3}$, A. Bertolin ${ }^{38}$,

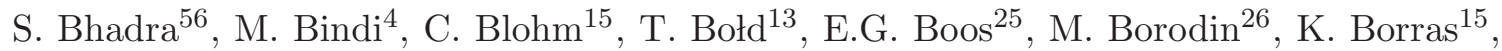
D. Boscherini ${ }^{3}$, D. Bot ${ }^{15}$, S.K. Boutle ${ }^{51}$, I. Brock ${ }^{5}$, E. Brownson ${ }^{55}$, R. Brugnera ${ }^{39}$, N. Brümmer ${ }^{36}$, A. Bruni ${ }^{3}$, G. Bruni ${ }^{3}$, B. Brzozowska ${ }^{52}$, P.J. Bussey ${ }^{20}$, J.M. Butterworth ${ }^{51}$, B. Bylsma ${ }^{36}$, A. Caldwell ${ }^{34}$, M. Capua ${ }^{8}$, R. Carlin ${ }^{39}$, C.D. Catterall ${ }^{56}$, S. Chekanov ${ }^{1}$, J. Chwastowski ${ }^{12}$, J. Ciborowski ${ }^{52, a i}$, R. Ciesielski ${ }^{15}$, L. Cifarelli ${ }^{4}$, F. Cindolo ${ }^{3}$, A. Contin ${ }^{4}$, A.M. Cooper-Sarkar ${ }^{37}$, N. Coppola ${ }^{15, j}$, M. Corradi ${ }^{3}$, F. Corriveau ${ }^{30}$, M. Costa $^{48}$, G. D'Agostini ${ }^{42}$, F. Dal Corso ${ }^{38}$, J. de Favereau ${ }^{28}$, J. del Peso ${ }^{29}$, R.K. Dementiev ${ }^{33}$, S. De Pasquale ${ }^{4, b}$, M. Derrick ${ }^{1}$, R.C.E. Devenish ${ }^{37}$, D. Dobur ${ }^{19}$, B.A. Dolgoshein ${ }^{32}$, A.T. Doyle ${ }^{20}$, V. Drugakov ${ }^{16}$, L.S. Durkin ${ }^{36}$, S. Dusini ${ }^{38}$, Y. Eisenberg ${ }^{54}$, P.F. Ermolov ${ }^{33, \dagger}$, A. Eskreys ${ }^{12}$, S. Fang ${ }^{15}$, S. Fazio ${ }^{8}$, J. Ferrando ${ }^{37}$, M.I. Ferrero ${ }^{48}$, J. Figiel ${ }^{12}$, M. Forrest ${ }^{20}$, B. Foster ${ }^{37}$, S. Fourletov ${ }^{50, a h}$, A. Galas ${ }^{12}$, E. Gallo ${ }^{17}$, A. Garfagnini ${ }^{39}$, A. Geiser ${ }^{15}$, I. Gialas ${ }^{21, u}$, L.K. Gladilin ${ }^{33}$, D. Gladkov ${ }^{32}$, C. Glasman ${ }^{29}$, O. Gogota ${ }^{26}$, Yu.A. Golubkov ${ }^{33}$, P. Göttlicher ${ }^{15, k}$, I. Grabowska-Bołd ${ }^{13}$, J. Grebenyuk ${ }^{15}$, I. Gregor ${ }^{15}$, G. Grigorescu ${ }^{35}$, G. Grzelak ${ }^{52}$, C. Gwenlan ${ }^{37, a a}$, T. Haas ${ }^{15}$, W. Hain ${ }^{15}$, R. Hamatsu 47 , J.C. Hart ${ }^{43}$, H. Hartmann ${ }^{5}$, G. Hartner ${ }^{56}$, E. Hilger ${ }^{5}$, D. Hochman ${ }^{54}$, U. Holm ${ }^{22}$, R. Hori ${ }^{46}$, K. Horton ${ }^{37, a b}$, A. Hüttmann ${ }^{15}$, G. Iacobucci ${ }^{3}$, Z.A. Ibrahim ${ }^{10}$, Y. Iga ${ }^{41}$, R. Ingbir ${ }^{44}$, M. Ishitsuka ${ }^{45}$, H.-P. Jakob ${ }^{5}$, F. Januschek ${ }^{15}$, M. Jimenez ${ }^{29}$, T.W. Jones ${ }^{51}$, M. Jüngst ${ }^{5}$, I. Kadenko ${ }^{26}$, B. Kahle ${ }^{15}$, B. Kamaluddin ${ }^{10}$, S. Kananov ${ }^{44}$, T. Kanno ${ }^{45}$, U. Karshon ${ }^{54}$, F. Karstens ${ }^{19}$, I.I. Katkov ${ }^{15, l}$, M. Kaur ${ }^{7}$, P. Kaur ${ }^{7, d}$, A. Keramidas ${ }^{35}$, L.A. Khein ${ }^{33}$, 
J.Y. Kim ${ }^{9, f}$, D. Kisielewska ${ }^{13}$, S. Kitamura ${ }^{47, a e}$, R. Klanner ${ }^{22}$, U. Klein ${ }^{15, m}$, E. Koffeman ${ }^{35}$, D. Kollar ${ }^{34}$, P. Kooijman ${ }^{35}$, Ie. Korol ${ }^{26}$, I.A. Korzhavina ${ }^{33}$, A. Kotański ${ }^{14, h}$, U. Kötz ${ }^{15}$, H. Kowalski ${ }^{15}$, P. Kulinski ${ }^{52}$, O. Kuprash ${ }^{26}$, M. Kuze ${ }^{45}$, V.A. Kuzmin ${ }^{33}$, A. Lee ${ }^{36}$, B.B. Levchenko ${ }^{33, z}$, A. Levy ${ }^{44}$, V. Libov $^{15}$, S. Limentani ${ }^{39}$, T.Y. Ling ${ }^{36}$, M. Lisovyi ${ }^{15}$, E. Lobodzinska ${ }^{15}$, W. Lohmann ${ }^{16}$, B. Löhr ${ }^{15}$, E. Lohrmann ${ }^{22}$, J.H. Loizides ${ }^{51}$, K.R. Long ${ }^{23}$, A. Longhin ${ }^{38}$, D. Lontkovskyi ${ }^{26}$, J. Lukasik ${ }^{13, g}$, O.Yu. Lukina ${ }^{33}$, P. Lużniak ${ }^{52, a j}$, J. Maeda ${ }^{45}$, S. Magill ${ }^{1}$, I. Makarenko ${ }^{26}$, J. Malka ${ }^{52, a j}$, R. Mankel ${ }^{15, n}$, A. Margotti ${ }^{3}$, G. Marini ${ }^{42}$, J.F. Martin ${ }^{50}$, A. Mastroberardino ${ }^{8}$, T. Matsumoto ${ }^{24, v}$, M.C.K. Mattingly ${ }^{2}$, I.-A. Melzer-Pellmann ${ }^{15}$, S. Miglioranzi ${ }^{15, o}$, F. Mohamad Idris ${ }^{10}$, V. Monaco ${ }^{48}$,

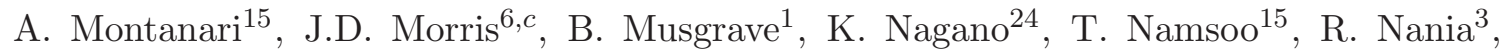
D. Nicholass ${ }^{1, a}$, A. Nigro ${ }^{42}$, Y. Ning ${ }^{11}$, U. Noor ${ }^{56}$, D. Notz $^{15}$, R.J. Nowak ${ }^{52}$, A.E. NuncioQuiroz $^{5}$, B.Y. $\mathrm{Oh}^{40}$, N. Okazaki ${ }^{46}$, K. Oliver ${ }^{37}$, K. Olkiewicz ${ }^{12}$, Yu. Onishchuk ${ }^{26}$, O. Ota ${ }^{47, a f}$, K. Papageorgiu ${ }^{21}$, A. Parenti ${ }^{15}$, E. Paul ${ }^{5}$, J.M. Pawlak ${ }^{52}$, B. Pawlik ${ }^{12}$, P. G. Pelfer ${ }^{18}$, A. Pellegrino ${ }^{35}$, W. Perlanski ${ }^{52, a j}$, H. Perrey ${ }^{22}$, K. Piotrzkowski ${ }^{28}$, P. Plucinski ${ }^{53, a k}$, N.S. Pokrovskiy ${ }^{25}$, A. Polini ${ }^{3}$, A.S. Proskuryakov ${ }^{33}$, M. Przybycień $^{13}$, A. Raval ${ }^{15}$, D.D. Reeder ${ }^{55}$, B. Reisert ${ }^{34}$, Z. Ren ${ }^{11}$, J. Repond ${ }^{1}$, Y.D. Ri ${ }^{47, a g}$, A. Robertson ${ }^{37}$, P. Roloff ${ }^{15}$, E. Ron ${ }^{29}$, I. Rubinsky ${ }^{15}$, M. Ruspa ${ }^{49}$, R. Sacchi ${ }^{48}$, A. Salii ${ }^{26}$, U. Samson ${ }^{5}$, G. Sartorelli ${ }^{4}$, A.A. Savin ${ }^{55}$, D.H. Saxon ${ }^{20}$, M. Schioppa ${ }^{8}$, S. Schlenstedt ${ }^{16}$, P. Schleper ${ }^{22}$, W.B. Schmidke ${ }^{34}$, U. Schneekloth ${ }^{15}$, V. Schönberg ${ }^{5}$, T. Schörner-Sadenius ${ }^{22}$, J. Schwartz ${ }^{30}$, F. Sciulli1 ${ }^{11}$, L.M. Shcheglova ${ }^{33}$, R. Shehzadi ${ }^{5}$, S. Shimizu ${ }^{46, o}$, I. Singh ${ }^{7, d}$, I.O. Skillicorn ${ }^{20}$, W. Słomiński ${ }^{14, i}$, W.H. Smith ${ }^{55}$, V. Sola ${ }^{48}$, A. Solano ${ }^{48}$, A. Solomin ${ }^{6}$, D. $\mathrm{Son}^{27}$, V. Sosnovtsev ${ }^{32}$, A. Spiridonov ${ }^{15, p}, \mathrm{H} . \mathrm{Stadie}^{22}$, L. Stanco ${ }^{38}$, A. Stern ${ }^{44}$, T.P. Stewart ${ }^{50}$, A. Stifutkin ${ }^{32}$, P. Stopa ${ }^{12}$, S. Suchkov ${ }^{32}$, G. Susinno ${ }^{8}$, L. Suszycki ${ }^{13}$, J. Sztuk ${ }^{22}$, D. Szuba ${ }^{15, q}$, J. Szuba ${ }^{15, r}$, A.D. Tapper ${ }^{23}$, E. Tassi ${ }^{8, e}$, J. Terrón ${ }^{29}$, T. Theedt ${ }^{15}$, H. Tiecke ${ }^{35}$, K. Tokushuku ${ }^{24, w}$, O. Tomalak ${ }^{26}$, J. Tomaszewska ${ }^{15, s}$, T. Tsurugai ${ }^{31}$,

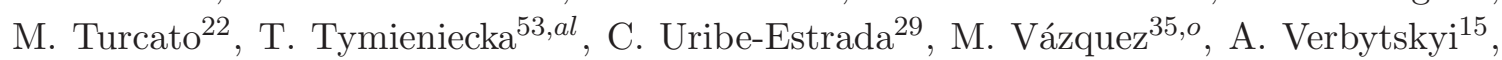
V. Viazlo ${ }^{26}$, N.N. Vlasov ${ }^{19, t}$, O. Volynets $^{26}$, R. Walczak ${ }^{37}$, W.A.T. Wan Abdullah ${ }^{10}$, J.J. Whitmore ${ }^{40, a c}$, J. Whyte ${ }^{56}$, L. Wiggers ${ }^{35}$, M. Wing ${ }^{51}$, M. Wlasenko ${ }^{5}$, G. Wolf ${ }^{15}$, H. Wolfe ${ }^{55}$, K. Wrona ${ }^{15}$, A.G. Yagües-Molina ${ }^{15}$, S. Yamada ${ }^{24}$, Y. Yamazaki ${ }^{24, x}$, R. Yoshida ${ }^{1}$, C. Youngman ${ }^{15}$, A.F. Żarnecki ${ }^{52}$, L. Zawiejski ${ }^{12}$, O. Zenaiev ${ }^{26}$, W. Zeuner ${ }^{15, n}$, B.O. Zhautykov ${ }^{25}$, N. Zhmak ${ }^{26, y}$, C. Zhou ${ }^{30}$, A. Zichichi ${ }^{4}$, M. Zolko ${ }^{26}$, D.S. Zotkin ${ }^{33}$

\footnotetext{
1 Argonne National Laboratory, Argonne, Illinois 60439-4815, USA A

2 Andrews University, Berrien Springs, Michigan 49104-0380, USA

3 INFN Bologna, Bologna, Italy ${ }^{B}$

4 University and INFN Bologna, Bologna, Italy ${ }^{B}$

5 Physikalisches Institut der Universität Bonn, Bonn, Germany ${ }^{C}$

$6 \quad$ H.H. Wills Physics Laboratory, University of Bristol, Bristol, United Kingdom ${ }^{D}$

$7 \quad$ Panjab University, Department of Physics, Chandigarh, India

8 Calabria University, Physics Department and INFN, Cosenza, Italy ${ }^{B}$

9 Chonnam National University, Kwangju, South Korea

10 Jabatan Fizik, Universiti Malaya, 50603 Kuala Lumpur, Malaysia ${ }^{E}$
} 

USA $F$ The Henryk Niewodniczanski Institute of Nuclear Physics, Polish Academy of Sciences, Cracow, Poland ${ }^{G}$

13 Faculty of Physics and Applied Computer Science, AGH-University of Science and Technology, Cracow, Poland ${ }^{H}$ 

project (2009-2010)

I supported by the Japanese Ministry of Education, Culture, Sports, Science and Technology (MEXT) and its grants for Scientific Research

$J$ supported by the Korean Ministry of Education and Korea Science and Engineering Foundation

$K$ supported by FNRS and its associated funds (IISN and FRIA) and by an InterUniversity Attraction Poles Programme subsidised by the Belgian Federal Science Policy Office

Rutherford Appleton Laboratory, Chilton, Didcot, Oxon, United Kingdom ${ }^{D}$ Raymond and Beverly Sackler Faculty of Exact Sciences, School of Physics, Tel Aviv University, Tel Aviv, Israel ${ }^{Q}$ Department of Physics, Tokyo Institute of Technology, Tokyo, Japan ${ }^{I}$ Department of Physics, University of Tokyo, Tokyo, Japan ${ }^{I}$

Tokyo Metropolitan University, Department of Physics, Tokyo, Japan ${ }^{I}$ Università di Torino and INFN, Torino, Italy ${ }^{B}$

Università del Piemonte Orientale, Novara, and INFN, Torino, Italy ${ }^{B}$

Department of Physics, University of Toronto, Toronto, Ontario, Canada M5S $1 A 7^{M}$

Kingdom ${ }^{D}$

Warsaw University, Institute of Experimental Physics, Warsaw, Poland Institute for Nuclear Studies, Warsaw, Poland

Department of Particle Physics, Weizmann Institute, Rehovot, Israel ${ }^{R}$

Department of Physics, University of Wisconsin, Madison, Wisconsin 53706, USA ${ }^{A}$

Department of Physics, York University, Ontario, Canada M3J 1Pз ${ }^{M}$

supported by the US Department of Energy

supported by the Italian National Institute for Nuclear Physics (INFN) under contract Nos. 05 HZ6PDA, 05 HZ6GUA, 05 HZ6VFA and 05 HZ4KHA supported by the Science and Technology Facilities Council, UK supported by an FRGS grant from the Malaysian government supported by the US National Science Foundation. Any opinion, findings and conclusions or recommendations expressed in this material are those of the authors and do not necessarily reflect the views of the National Science Foundation. supported by the Polish State Committee for Scientific Research, project No. DESY/256/2006 - 154/DES/2006/03

$H$ supported by the Polish Ministry of Science and Higher Education as a scientific 
$L \quad$ supported by the Spanish Ministry of Education and Science through funds provided by CICYT

$M \quad$ supported by the Natural Sciences and Engineering Research Council of Canada (NSERC)

$N$ partially supported by the German Federal Ministry for Education and Research (BMBF)

$O \quad$ supported by RF Presidential grant N 1456.2008.2 for the leading scientific schools and by the Russian Ministry of Education and Science through its grant for Scientific Research on High Energy Physics

$P \quad$ supported by the Netherlands Foundation for Research on Matter (FOM)

$Q \quad$ supported by the Israel Science Foundation

$R$ supported in part by the MINERVA Gesellschaft für Forschung $\mathrm{GmbH}$, the Israel Science Foundation (grant No. 293/02-11.2) and the US-Israel Binational Science Foundation

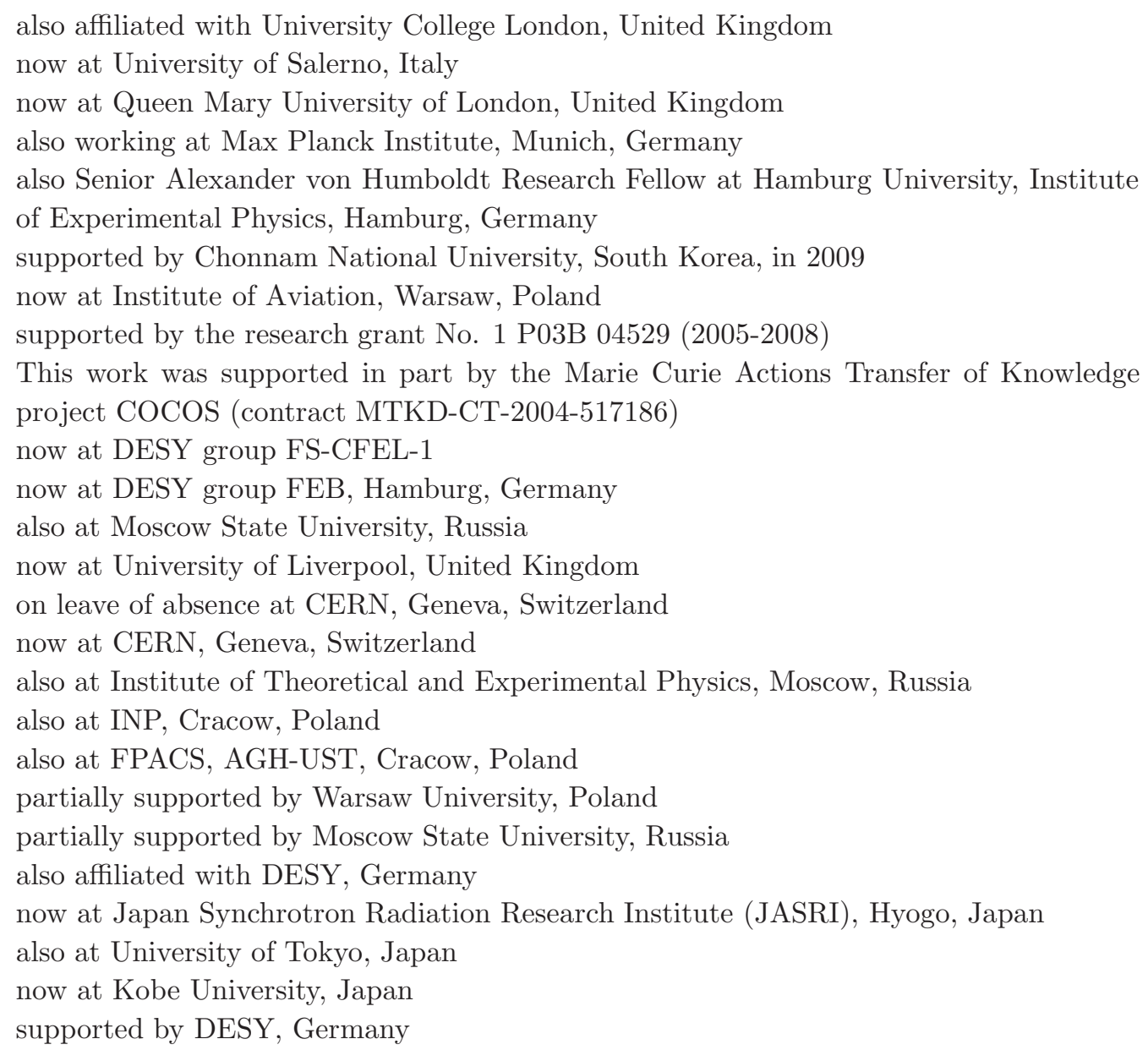


$z \quad$ partially supported by Russian Foundation for Basic Research grant

No. 05-02-39028-NSFC-a

$\dagger$ deceased

aa $\quad$ STFC Advanced Fellow

ab nee Korcsak-Gorzo

ac This material was based on work supported by the National Science Foundation, while working at the Foundation.

ad also at Max Planck Institute, Munich, Germany, Alexander von Humboldt Research Award

ae now at Nihon Institute of Medical Science, Japan

af now at SunMelx Co. Ltd., Tokyo, Japan

ag now at Osaka University, Osaka, Japan

ah now at University of Bonn, Germany

ai also at Łódź University, Poland

aj member of Łódź University, Poland

ak now at Lund University, Lund, Sweden

al also at University of Podlasie, Siedlce, Poland 